\title{
Self-Organizing Adaptive Wavelet Backstepping Control Research for AC Servo System
}

\author{
Run-min Hou, Yuan-long Hou, Qiang Gao, and Chao Wang \\ Department of Mechanical Engineering, Nanjing University of Science and Technology, Nanjing 210094, China \\ Correspondence should be addressed to Run-min Hou; 187189579@qq.com
}

Received 8 September 2015; Revised 27 October 2015; Accepted 28 October 2015

Academic Editor: Vadim V. Silberschmidt

Copyright (C) 2016 Run-min Hou et al. This is an open access article distributed under the Creative Commons Attribution License, which permits unrestricted use, distribution, and reproduction in any medium, provided the original work is properly cited.

\begin{abstract}
A novel self-organizing adaptive wavelet cerebellar model articulation controller backstepping (SOWCB) control is proposed, aiming at some nonlinear and uncertain factors that caused difficulties in controlling the AC servo system. This controller consists of self-organizing wavelet cerebellar model articulation controller (CMAC) and robust compensator. It absorbs fast learning and precise approaching advantage of self-organizing wavelet CMAC to mimic a backstepping controller, and then robust compensator is added to inhibit influence of the uncertainties on system performance effectively and realize high accuracy position tracking for AC servo system. Moreover, the stability of the control system can be guaranteed by using Lyapunov method. The results of the simulation and the prototype test prove that the proposed approach can improve the steady state performance and control accuracy and possess a strong robustness to both parameter perturbation and load disturbance.
\end{abstract}

\section{Introduction}

With the advancement of technology, AC servo systems have become more and more widely utilized. For a servo driving system, the control system is required to have both a strong steady-stage and dynamic performance, and it is necessary to build a precise dynamic model of the system for conducting the analysis, simulation, and control of an AC servo system. As a controlled object, the dynamic mathematical model of an AC motor is a complex system, which is characterized by a heavy varying-load, slow time variation, nonlinearity, and uncertain disturbance. Thus, the practical intelligent control strategy has become a focus in the field of servo system control.

Zhou and Zhu $[1,2]$ use nonlinear backstepping control method that can effectively realize the nonlinear decoupling of AC servo system and ensure the accuracy of the motor servo control at the same time; however, the algorithm requires accurate mathematical model of controlled object that is known; if the controlled object has uncertain amount of interference, the algorithm will not be able to achieve good control effect.
$\mathrm{Su}$ and $\mathrm{He}[3,4]$ for uncertain nonlinear system using backstepping control and learning control method of combining designed several adaptive learning controllers; the literature [5-7] uses wavelet neural network approach in the use of complex nonlinear term backstepping process; the control structure has been simplified and improved learning ability.

Cerebellar model articulation controller (CMAC) is modeled on the principle of the cerebellum control body movement established $[8,9]$. Peng and Lin $[10,11]$ use CMAC to replace the traditional neural network approximation to complex nonlinear system. The literature [12] studies a Gaussian basis function neural network, making its generalization ability enhanced. Lee et al. [13] propose a self-organizing genetic neural network algorithm; however the structure design method is too complicated and lacks online learning ability.

Based on the above analysis, this paper uses selforganizing adaptive wavelet cerebellar model articulation controller (SOWC) to online approximation of backstepping controller, using robust control to eliminate system uncertainties and approximation error; finally, simulation 


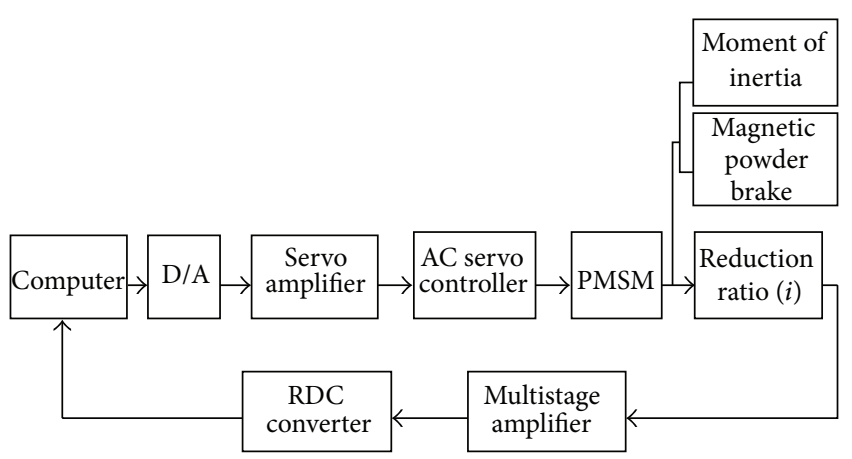

FIgURE 1: The structure diagram of AC servo system.

experiments of AC servo system and the prototype test can prove the effectiveness of the proposed method.

\section{Modeling AC Servo System}

The structure diagram of an AC servo system is presented in Figure 1, where the magnetic powder brake is the purpose of the change of load simulation system. Because this paper's main consideration is the system load changes caused by the nonlinear of the motor itself is correct, the system load changes brought nonlinear phase comparison that is very small, so the derivation of the model made the following assumptions:

(1) No saturation effect.

(2) Induction electromotive force that is sine wave shape; motor air-gap magnetic field distribution.

(3) Excluding the hysteresis and eddy current loss.

(4) No rotor excitation winding.

Based on the above assumptions, available under $d$ - $q$ axis two-phase static coordinate system mathematical model is the following:

$$
\begin{aligned}
& \dot{i}_{d}=-\frac{R}{L_{d}} i_{d}+\frac{L_{q}}{L_{d}} p i_{q} \omega_{r}+\frac{u_{d}}{L_{d}} \\
& \dot{i}_{q}=-\frac{R}{L_{q}} i_{q}+\frac{L_{d}}{L_{q}} p i_{d} \omega_{r}-\frac{\psi_{f}}{L_{q}} p \omega_{r}+\frac{u_{q}}{L_{q}}, \\
& \dot{\omega}_{r}=\frac{1}{J}\left(T_{e}-T_{L}-B \omega_{r}\right)
\end{aligned}
$$

where $u_{d}$ and $u_{q}$ are $d-q$ axis stator voltage component; $i_{d}$ and $i_{q}$ are $d-q$ axis stator current component; $L_{d}, L_{q}$ are $d-q$ axis stator inductor component; $\psi_{f}$ is flux linkage; $R$ is stator resistance; $P$ is pole-pairs; $T_{L}$ is the load disturbance torque; $T_{e}$ is electromagnetic torque; $J$ is the total inertia moment converted to the rotor; $B$ is the viscous friction coefficient; $\omega_{r}$ is the rotor velocity of motor; $i$ is the reduction ratio.

Use the method of vector control technology of $i_{d}=$ 0 to achieve linear resolve decoupling control. Mechanical equations can be derived by (1):

$$
J \dot{\omega}_{b}+B \omega_{b}+T_{L}=T_{e}
$$

where $\omega_{b}$ is the angular velocity of motor, $\omega_{r}=p \omega_{b}$; the equation of electromagnetic torque is shown as

$$
T_{e}=\frac{3}{2} p \psi_{f} i_{q}=K_{t} i_{q}
$$

where $K_{t}$ is torque constant.

Due to the current in the motor time constant being far smaller than the mechanical time constant, the current loop speed is faster than the response speed of the speed loop and position loop, so the current loop approximation can be simplified as a proportion function.

Let variable $x_{1}=\theta, x_{2}=\omega_{r}=\dot{\theta}$, put (3) into (2), and the state space equation of the speed control system can be rewritten as

$$
\begin{aligned}
& \dot{x}_{1}=x_{2} \\
& \dot{x}_{2}=-\frac{B}{J} x_{2}(t)+\frac{K_{t}}{J} i_{q}(t)+\left(-\frac{1}{J} T_{L}\right) .
\end{aligned}
$$

Equation (4) can be rewritten as

$$
\dot{x}_{2}=f(x) x_{2}(t)+g(x) i_{q}(t)+d(t)
$$

where $f(x)=-B / J ; g(x)=K_{t} / J ; d(t)=-T_{L} / J$; and $|d(t)| \leq$ $C$, where $C$ is a constant.

\section{Self-Organizing Wavelet CMAC Neural Networks}

Self-organizing wavelet CMAC network structure diagram is as shown in Figure 2.

The network consists of input space, store space, accepted domain space, weights of storage space, and output space [14]. The output expression is

$$
O=u_{\text {sowc }}(\mathbf{I}, \boldsymbol{\omega}, \mathbf{a}, \mathbf{b})=\sum_{k=1}^{n_{b}} \omega_{k} r_{k}\left(\mathbf{I}, \mathbf{a}_{k}, \mathbf{b}_{k}\right)
$$

where $n_{b}$ is layer node; $k=1,2, \ldots, n_{b} ; \omega_{k}$ is the output of weight memory at the $k$ node. $r_{k}$ is accepted space at the $k$ node; each accepted space layer has different wavelet functions; the $r_{k}$ can be shown as

$$
\begin{aligned}
r_{k}\left(\mathbf{I}, \mathbf{a}_{k}, \mathbf{b}_{k}\right)=\prod_{i=1}^{n_{a}} \mu_{i k} F_{i k} \\
\quad=-\prod_{i=1}^{n_{a}}\left(\frac{I_{i}-a_{i k}}{b_{i k}}\right) \exp \left(\frac{-\left(\left(I_{i}-a_{i k}\right) / b_{i k}\right)^{2}}{2}\right),
\end{aligned}
$$

where $a_{i k}$ is wavelet translated parameter and $b_{i k}$ is wavelet scaling parameter. 


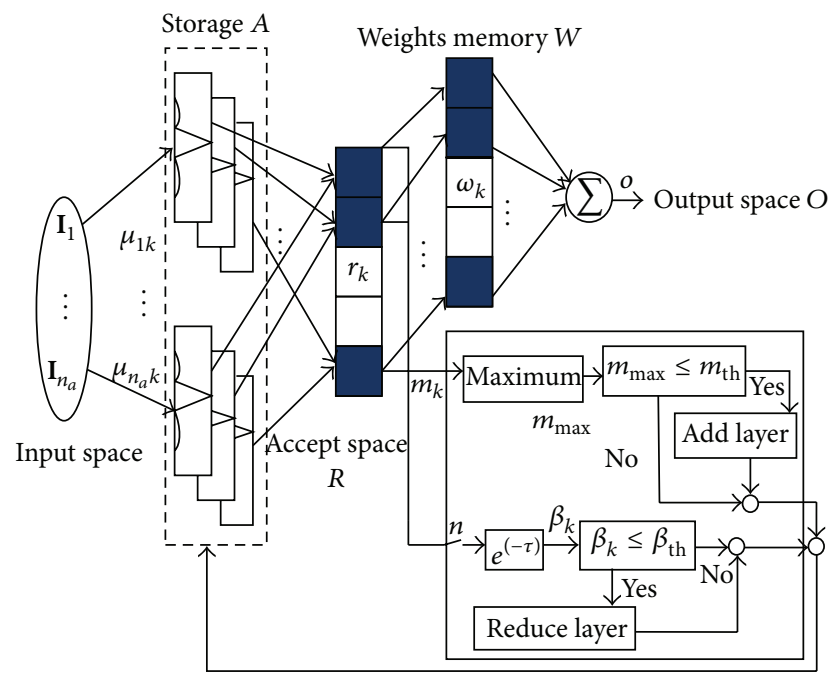

FIGURE 2: The structure of self-organizing wavelet CMAC neural networks.

For the convenience of deriving, the parameter of CMAC can be defined as

$$
\begin{aligned}
\mathbf{I} & =\left[I_{1}, \ldots, I_{n_{a}}\right]^{\mathrm{T}} \in \mathfrak{R}^{n_{a}} ; \\
\mathbf{r} & =\left[r_{1}, \ldots, r_{n_{b}}\right]^{\mathrm{T}} \in \mathfrak{R}^{n_{b}} ; \\
\boldsymbol{\omega} & =\left[\omega_{1}, \ldots, \omega_{n_{b}}\right]^{\mathrm{T}} \in \mathfrak{R}^{n_{b}} ; \\
\mathbf{a}_{k} & =\left[a_{1 k}, \ldots, a_{n_{a} k}\right]^{\mathrm{T}} \in \mathfrak{R}^{n_{a}} ; \\
\mathbf{a} & =\left[\mathbf{a}_{1}^{\mathrm{T}}, \ldots, \mathbf{a}_{n_{b}}^{\mathrm{T}}\right]^{\mathrm{T}} \in \mathfrak{R}^{n_{a} n_{b}} ; \\
\mathbf{b}_{k} & =\left[b_{1 k}, \ldots, b_{n_{a} k}\right]^{\mathrm{T}} \in \mathfrak{R}^{n_{a}} ; \\
\mathbf{b} & =\left[\mathbf{b}_{1}^{\mathrm{T}}, \ldots, \mathbf{b}_{n_{b}}^{\mathrm{T}}\right]^{\mathrm{T}} \in \mathfrak{R}^{n_{a} n_{b}} .
\end{aligned}
$$

3.1. Layer Node Increase. According to the size of the input to increase or decrease the number of nodes, if a new input is valued within the range of this family, the self-organizing cerebellar neural network will no longer produce new node; it will just change the weight [15].

Defined in the association storage space,

$$
m_{k}=r_{k}, \quad k=1,2, \ldots, n_{b}(t),
$$

where $m_{k}$ is measuring degrees of network at $k$ nodes. With the following theory to determine the number of nodes increases, it can be expressed as

$$
m_{\max }=\max _{1 \leq k \leq n_{b}} m_{k}
$$

Set $m_{\mathrm{th}} \in(0,1)$ as a predetermined minimum; if it is satisfied by $m_{\max } \leq m_{k}$, then a new node can be generated by

$$
n_{b}(t+1)=n_{b}(t)+1
$$

Translated and scaling parameter in the memory space and weight of the new generation are set to

$$
\begin{aligned}
& a_{i}^{\text {new }}=I_{i} ; \\
& b_{i}^{\text {new }}=b_{i} ; \\
& \omega^{\text {new }}=0,
\end{aligned}
$$

where $I_{i}$ is new input data and $b_{i}$ is a preset constant.

3.2. Layer Node Decrease. Consider the exponential function at $k$ node is

$$
\beta_{k}(t+1)= \begin{cases}\beta_{k}(t) \exp (-\tau), & \text { if } m_{k}(t)<n \\ \beta_{k}(t), & \text { if } m_{k}(t)>n,\end{cases}
$$

where $n$ is to reduce the threshold; $\tau$ is speed constant; $\beta_{k}(t)$ is the initial value of 1 at the first index $k$ layer, where $k=$ $1, \ldots, n_{b}(t)$; if $\beta_{k}$ is less than a given threshold $\beta_{\text {th }}$ in advance, the first $k$ node should be deleted. This means that, for an output data, if a node for the contribution of output is less than a set value, then this node should be deleted.

\section{Self-Organizing Wavelet Adaptive CMAC Backstepping Control}

4.1. Ideal Backstepping Control. In this paper, the control goal is to make the position of the system able to track the given trajectory asymptotically stable signal. For achieving this goal, assume $f(x), g(x)$, and $d(t)$ are known in (5); the steps of ideal backstepping method are as follows.

Step 1. Define position error:

$$
e_{1}=\theta_{d}-\theta
$$

where $\theta_{d}$ is input signal.

Define virtual control inputs:

$$
\alpha=c_{1} e_{1}+\dot{\theta}_{d},
$$

where $c_{1}$ is a constant, which is greater than 0 .

Step 2. Define

$$
e_{2}=\alpha-\dot{\theta}
$$

where $\dot{e}_{2}$ is

$$
\dot{e}_{2}=\dot{\alpha}-\ddot{\theta}=\dot{\alpha}-f(x)-g(x) u-d(t) .
$$

Step 3. Define Lyapunov function:

$$
V_{1}=\frac{1}{2} e_{1}^{2}+\frac{1}{2} e_{2}^{2}
$$

where

$$
\begin{aligned}
\dot{V}_{1} & =-c_{1} e_{1}^{2}+e_{2} e_{1}+e_{2} \dot{e}_{2} \\
& =-c_{1} e_{1}^{2}+e_{2}\left[e_{1}+\dot{\alpha}-f(x)-g(x) u-d(t)\right] .
\end{aligned}
$$


In order to make $\dot{V}_{1} \leq 0$, the ideal backstepping controller is designed for

$$
u_{\mathrm{IBC}}=\frac{1}{g(x)}\left[\dot{\alpha}-f(x)-d(t)+e_{1}+c_{2} e_{2}\right],
$$

where $c_{2}$ is a constant, which is greater than 0 .

Put (20) into (19); it can get

$$
\dot{V}_{1}=-c_{1} e_{1}^{2}-c_{2} e_{2}^{2} \leq 0 .
$$

Thus, the asymptotic stability of the system can be guaranteed by the design of the control law.

4.2. SOWCB Design. Because the system is characterized by a heavy varying-load, slow time variation, nonlinearity, and uncertain disturbance, the ideal backstepping control algorithm is hard to get directly by (20). In order to solve this problem, in this paper, by using self-organizing wavelet adaptive CMAC control for an ideal to approximate backstepping controller, robust control is used to eliminate disturbance and approximation errors in the system; the control output can be shown as

$$
u_{\mathrm{SOWCB}}=u_{\mathrm{SOWC}}+u_{R}
$$

where $u_{\text {SowC }}$ is self-organizing wavelet CMAC control; $u_{R}$ is robust controller; $u_{\text {SOWCB }}$ is SOWCB control.

Assume the optimal SOWC controller for an ideal to approximate backstepping controller, which is shown as

$$
u_{\mathrm{IBC}}^{\prime}=u_{\text {SOWC }}^{\prime}\left(\mathbf{I}, \mathbf{w}^{\prime}, \mathbf{a}^{\prime}, \mathbf{b}^{\prime}\right)=\boldsymbol{\omega}^{\prime \mathrm{T}} \mathbf{r}^{\prime}\left(\mathbf{a}^{\prime}, \mathbf{b}^{\prime}\right)+\varepsilon,
$$

where $\varepsilon$ is the minimum reconstruction error; $\boldsymbol{\omega}^{\prime}, \mathbf{r}^{\prime}, \mathbf{a}^{\prime}$, and $\mathbf{b}^{\prime}$ represent optimum parameter by $\boldsymbol{\omega}, \mathbf{r}, \mathbf{a}$, and $\mathbf{b}$; the optimal node is divided into two parts: the first part is the activation layer, including $n_{b}$ node; the second part is the inactive parts, which include $n_{b}^{\prime}-n_{b}$. The optimal parameters $\boldsymbol{\omega}^{\prime}, \mathbf{r}^{\prime}, \mathbf{a}^{\prime}$, and $\mathbf{b}^{\prime}$ can be divided into two parts, which is shown as

$$
\begin{aligned}
\boldsymbol{\omega}^{\prime} & =\left[\begin{array}{c}
\boldsymbol{\omega}_{e}^{\prime} \\
\boldsymbol{\omega}_{i}^{\prime}
\end{array}\right] ; \\
\mathbf{a}^{\prime} & =\left[\begin{array}{c}
\mathbf{a}_{e}^{\prime} \\
\mathbf{a}_{i}^{\prime}
\end{array}\right] ; \\
\mathbf{b}^{\prime} & =\left[\begin{array}{l}
\mathbf{b}_{e}^{\prime} \\
\mathbf{b}_{i}^{\prime}
\end{array}\right] ; \\
\mathbf{r}^{\prime} & =\left[\begin{array}{c}
\mathbf{r}_{e}^{\prime} \\
\mathbf{r}_{i}^{\prime}
\end{array}\right],
\end{aligned}
$$

where $\boldsymbol{\omega}_{e}^{\prime}, \mathbf{r}_{e}^{\prime}, \mathbf{a}_{e}^{\prime}$, and $\mathbf{b}_{e}^{\prime}$ are active part, respectively; $\boldsymbol{\omega}_{i}^{\prime}, \mathbf{r}_{i}^{\prime}, \mathbf{a}_{i}^{\prime}$, and $\mathbf{b}_{i}^{\prime}$ are inactive part, respectively.

Because the optimal SOWC is not easy to get, thus, get $\widehat{u}_{\text {sowc }}$ to approximate optimal value online. The control law equation (22) can be rewritten as

$$
\begin{aligned}
u_{\mathrm{SOWCB}} & =\widehat{u}_{\text {SOWC }}\left(\mathbf{I}, \widehat{\boldsymbol{\omega}}_{e}, \widehat{\mathbf{a}}_{e}, \widehat{\mathbf{b}}_{e}\right)+u_{R} \\
& =\widehat{\boldsymbol{\omega}}_{e}^{\mathrm{T}} \widehat{\mathbf{r}}_{e}\left(\widehat{\mathbf{a}}_{e}, \widehat{\mathbf{b}}_{e}\right)+u_{R},
\end{aligned}
$$

where $\widehat{\boldsymbol{\omega}}_{e}, \widehat{\mathbf{a}}_{e}, \widehat{\mathbf{b}}_{e}$, and $\widehat{\mathbf{r}}_{e}$, respectively, are the optimal estimates of the parameters $\boldsymbol{\omega}_{e}^{\prime}, \mathbf{a}_{e}^{\prime}, \mathbf{b}_{e}^{\prime}$, and $\mathbf{r}_{e}^{\prime}$.

Put (25) into (23); the estimate error $\tilde{u}$ is shown as

$$
\begin{aligned}
\widetilde{u} & =\boldsymbol{\omega}_{e}^{\prime \mathrm{T}} \mathbf{r}_{e}^{\prime}+\boldsymbol{\omega}_{i}^{\prime \mathrm{T}} \mathbf{r}_{i}^{\prime}+\varepsilon-\widehat{\boldsymbol{\omega}}_{e}^{\mathrm{T}} \widehat{\mathbf{r}}_{e}-u_{R} \\
& =\widetilde{\boldsymbol{\omega}}_{e}^{\mathrm{T}} \mathbf{r}_{e}^{\prime}+\widehat{\boldsymbol{\omega}}_{e}^{\mathrm{T}} \widetilde{\mathbf{r}}_{e}+\boldsymbol{\omega}_{i}^{\prime \mathrm{T}} \mathbf{r}_{i}^{\prime}+\varepsilon-u_{R},
\end{aligned}
$$

where $\widetilde{\boldsymbol{\omega}}_{e}$ is $\boldsymbol{\omega}_{e}^{\prime}-\widehat{\boldsymbol{\omega}}_{e}$ and $\widetilde{\mathbf{r}}_{e}$ is $\mathbf{r}_{e}^{\prime}-\widehat{\mathbf{r}}_{e}$.

Wavelet function becomes a part of the linear form [16], and, according to the Taylor series expansion, the $\widetilde{\mathbf{r}}_{e}$ can be shown as

$$
\begin{aligned}
\widetilde{\mathbf{r}}_{e}=\left[\begin{array}{c}
\tilde{r}_{1} \\
\vdots \\
\tilde{r}_{k} \\
\vdots \\
\tilde{r}_{n_{b}}
\end{array}\right] \\
=\left[\begin{array}{c}
\left(\frac{\partial r_{1}}{\partial \mathbf{a}_{e}}\right)^{\mathrm{T}} \\
\vdots \\
\left(\frac{\partial r_{k}}{\partial \mathbf{a}_{e}}\right)^{\mathrm{T}} \\
\vdots \\
\left(\frac{\partial r_{n_{b}}}{\partial \mathbf{a}_{e}}\right)^{\mathrm{T}}
\end{array}\right]\left(\mathbf{a}_{e}^{\prime}-\widehat{\mathbf{a}}_{e}\right)+\left[\begin{array}{c}
\left(\frac{\partial r_{1}}{\partial \mathbf{b}_{e}}\right)^{\mathrm{T}} \\
\vdots \\
\left(\frac{\partial r_{k}}{\partial \mathbf{b}_{e}}\right)^{\mathrm{T}} \\
\vdots \\
\left(\frac{\partial r_{n_{b}}}{\partial \mathbf{b}_{e}}\right)^{\mathrm{T}}
\end{array}\right]\left(\mathbf{b}_{e}^{\prime}-\widehat{\mathbf{b}}_{e}\right) \\
+\mathbf{O}_{t}=\mathbf{r}_{a}^{\mathrm{T}} \widetilde{\mathbf{a}}_{e}+\mathbf{r}_{b}^{\mathrm{T}} \widetilde{\mathbf{b}}_{e}+\mathbf{O}_{t},
\end{aligned}
$$

where $\widetilde{\mathbf{a}}_{e}$ is $\mathbf{a}_{e}^{\prime}-\widehat{\mathbf{a}}_{e} ; \widetilde{\mathbf{b}}_{e}$ is $\mathbf{b}_{e}^{\prime}-\widehat{\mathbf{b}}_{e} ; \mathbf{O}_{t}$ is vector of the higher order term.

Equation (27) of $\partial r_{k} / \partial \mathbf{a}_{e}$ and $\partial r_{k} / \partial \mathbf{b}_{e}$ can be defined as

$$
\begin{aligned}
& {\left[\frac{\partial r_{k}}{\partial \mathbf{a}_{e}}\right]^{\mathrm{T}}=[\underbrace{0, \ldots, 0}_{(k-1) \times n_{a}}, \frac{\partial r_{k}}{\partial a_{1 k}}, \ldots, \frac{\partial r_{k}}{\partial a_{n_{a} k}}, \underbrace{0, \ldots, 0}_{\left(n_{b}-k\right) \times n_{a}},],} \\
& {\left[\frac{\partial r_{k}}{\partial \mathbf{b}_{e}}\right]^{\mathrm{T}}=[\underbrace{0, \ldots, 0}_{(k-1) \times n_{a}}, \frac{\partial r_{k}}{\partial b_{1 k}}, \ldots, \frac{\partial r_{k}}{\partial b_{n_{a} k}}, \underbrace{0, \ldots, 0}_{\left(n_{b}-k\right) \times n_{a}},] .}
\end{aligned}
$$

Equation (27) can be rewritten as

$$
\mathbf{r}_{e}^{\prime}=\widehat{\mathbf{r}}_{e}+\mathbf{r}_{a}^{\mathrm{T}} \widetilde{\mathbf{a}}_{e}+\mathbf{r}_{b}^{\mathrm{T}} \widetilde{\mathbf{b}}_{e}+\mathbf{O}_{t} .
$$

Put (27) and (29) into (26); it can get

$$
\begin{aligned}
\widetilde{u}= & \widetilde{\boldsymbol{w}}_{e}^{\mathrm{T}}\left(\widehat{\mathbf{r}}_{e}+\mathbf{r}_{a}^{\mathrm{T}} \widetilde{\mathbf{a}}_{e}+\mathbf{r}_{b}^{\mathrm{T}} \widetilde{\mathbf{b}}_{e}+\mathbf{O}_{t}\right) \\
& +\widehat{\boldsymbol{\omega}}_{e}^{\mathrm{T}}\left(\mathbf{r}_{a}^{\mathrm{T}} \widetilde{\mathbf{a}}_{e}+\mathbf{r}_{b}^{\mathrm{T}} \widetilde{\mathbf{b}}_{e}+\mathbf{O}_{t}\right)+\boldsymbol{\omega}_{i}^{\prime \mathrm{T}} \mathbf{r}_{i}^{\prime}+\varepsilon-u_{R} \\
= & \widetilde{\boldsymbol{\omega}}_{e}^{\mathrm{T}} \widehat{\mathbf{r}}_{e}+\widehat{\boldsymbol{\omega}}_{e}^{\mathrm{T}}\left(\mathbf{r}_{a}^{\mathrm{T}} \widetilde{\mathbf{a}}_{e}+\mathbf{r}_{b}^{\mathrm{T}} \widetilde{\mathbf{b}}_{e}\right)+\xi-u_{R},
\end{aligned}
$$


where the approximate error $\xi$ is shown as

$$
\xi=\widetilde{\boldsymbol{\omega}}_{e}^{\mathrm{T}} \mathbf{r}_{a}^{\mathrm{T}} \widetilde{\mathbf{a}}_{e}+\widetilde{\boldsymbol{\omega}}_{e}^{\mathrm{T}} \mathbf{r}_{b}^{\mathrm{T}} \widetilde{\mathbf{b}}_{e}+\boldsymbol{\omega}_{e}^{\prime \mathrm{T}} \mathbf{O}_{t}+\boldsymbol{\omega}_{i}^{\prime \mathrm{T}} \mathbf{r}_{i}^{\prime}+\varepsilon .
$$

Substitute (20) and (30) into (17); it can get

$$
\begin{aligned}
\dot{e}_{2}(t)= & \dot{\alpha}(t)-f(x, t)-g(x, t)-d(x, t) \\
= & g(x)\left(u_{\mathrm{IBC}}-u\right)-c_{2} e_{2}(t)-e_{1}(t) \\
= & g(x) \tilde{u}-c_{2} e_{2}(t)-e_{1}(t) \\
= & g(x)\left[\widetilde{\boldsymbol{\omega}}_{e}^{\mathrm{T}} \widehat{\mathbf{r}}_{e}+\widehat{\boldsymbol{\omega}}_{e}^{\mathrm{T}}\left(\mathbf{r}_{a}^{\mathrm{T}} \widetilde{\mathbf{a}}_{e}+\mathbf{r}_{b}^{\mathrm{T}} \widetilde{\mathbf{b}}_{e}\right)+\xi-u_{R}\right] \\
& -c_{2} e_{2}(t)-e_{1}(t) .
\end{aligned}
$$

To set up the system of adaptive parameter,

$$
\begin{aligned}
& \dot{\hat{\boldsymbol{\omega}}}_{e}=\eta_{1} e_{2}(t) \widehat{\mathbf{r}}_{e}, \\
& \dot{\widehat{\mathbf{a}}}_{e}=\eta_{2} e_{2}(t) \mathbf{r}_{a} \widehat{\boldsymbol{\omega}}_{e}, \\
& \dot{\widehat{\mathbf{b}}}_{e}=\eta_{3} e_{2}(t) \mathbf{r}_{b} \widehat{\boldsymbol{\omega}}_{e},
\end{aligned}
$$

where $\eta_{1}, \eta_{2}, \eta_{3}$ are positive constants. Self-organizing wavelet adaptive CMAC backstepping control is shown in Figure 3.

The robust compensator is designed as $[10,17]$

$$
u_{R}=\frac{\rho^{2}+1}{2 \rho^{2}} e_{2}(t),
$$

where $\rho$ is a positive constant.

Define Lyapunov function

$$
V_{2}=V_{1}+\frac{1}{2 \eta_{1}} \widetilde{\boldsymbol{\omega}}_{e}^{\mathrm{T}} \widetilde{\boldsymbol{\omega}}_{e}+\frac{1}{2 \eta_{2}} \widetilde{\mathbf{a}}_{e}^{\mathrm{T}} \widetilde{\mathbf{a}}_{e}+\frac{1}{2 \eta_{3}} \widetilde{\mathbf{b}}_{e}^{\mathrm{T}} \widetilde{\mathbf{b}}_{e}
$$

According to (37) and (32), the $\dot{V}_{2}$ can be shown as

$$
\begin{aligned}
& \dot{V}_{2}=\dot{V}_{1}+\frac{1}{\eta_{1}} \widetilde{\boldsymbol{\omega}}_{e}^{\mathrm{T}} \dot{\overrightarrow{\boldsymbol{\omega}}}_{e}+\frac{1}{\eta_{2}} \widetilde{\mathbf{a}}_{e}^{\mathrm{T}} \dot{\overrightarrow{\mathbf{a}}}_{e}+\frac{1}{\eta_{3}} \widetilde{\mathbf{b}}_{e}^{\mathrm{T}} \dot{\widetilde{\mathbf{b}}}_{e}=-c_{1} e_{1}^{2}(t) \\
& +e_{1}(t) e_{2}(t)+e_{2}(t) \dot{e}_{2}(t)+\frac{1}{\eta_{1}} \widetilde{\boldsymbol{\omega}}_{e}^{\mathrm{T}} \dot{\boldsymbol{\omega}}_{e}+\frac{1}{\eta_{2}} \widetilde{\mathbf{a}}_{e}^{\mathrm{T}} \dot{\overrightarrow{\mathbf{a}}}_{e} \\
& +\frac{1}{\eta_{3}} \widetilde{\mathbf{b}}_{e}^{\mathrm{T}} \dot{\widetilde{\mathbf{b}}}_{e}=-c_{1} e_{1}^{2}(t)+e_{1}(t) e_{2}(t)+e_{2}(t) \\
& \cdot\left[\left(\widetilde{\boldsymbol{\omega}}_{e}^{\mathrm{T}} \widehat{\mathbf{r}}_{e}+\widehat{\boldsymbol{w}}_{e}^{\mathrm{T}}\left(\mathbf{r}_{a}^{\mathrm{T}} \widetilde{\mathbf{a}}_{e}+\mathbf{r}_{b}^{\mathrm{T}} \widetilde{\mathbf{b}}_{e}\right)+\xi-u_{R}\right)\right. \\
& \left.-c_{2} e_{2}(t)-e_{1}(t)\right]+\frac{1}{\eta_{1}} \widetilde{\boldsymbol{\omega}}_{e}^{\mathrm{T}} \dot{\tilde{\boldsymbol{\omega}}}_{e}+\frac{1}{\eta_{2}} \widetilde{\mathbf{a}}_{e}^{\mathrm{T}} \dot{\overrightarrow{\mathbf{a}}}_{e}+\frac{1}{\eta_{3}} \widetilde{\mathbf{b}}_{e}^{\mathrm{T}} \dot{\widetilde{\mathbf{b}}}_{e} \\
& =-c_{1} e_{1}^{2}(t)-c_{2} e_{2}^{2}(t)+\widetilde{\boldsymbol{\omega}}_{e}^{\mathrm{T}}\left[e_{2}(t) \widehat{\mathbf{r}}_{e}-\frac{1}{\eta_{1}} \dot{\widehat{\boldsymbol{\omega}}}_{e}\right] \\
& +\widetilde{\mathbf{a}}_{e}^{\mathrm{T}}\left[e_{2}(t) \widehat{\mathbf{r}}_{a} \widehat{\boldsymbol{w}}_{e}-\frac{1}{\eta_{2}} \dot{\widehat{\mathbf{a}}}_{e}\right]+\widetilde{\mathbf{b}}_{e}^{\mathrm{T}}\left[e_{2}(t) \widehat{\mathbf{r}}_{b} \widehat{\boldsymbol{w}}_{e}\right. \\
& \left.-\frac{1}{\eta_{3}} \dot{\hat{\mathbf{b}}}_{e}\right]+e_{2}(t)\left[\xi-u_{R}\right]
\end{aligned}
$$

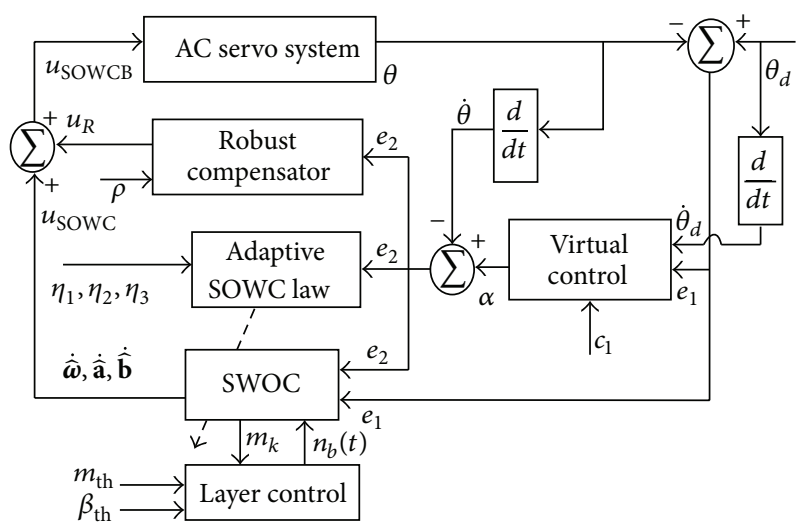

FIGURE 3: Self-organizing wavelet CMAC adaptive backstepping control.

Put (33) (36) into (38); it can get

$$
\begin{aligned}
\dot{V}_{2}= & -c_{1} e_{1}^{2}(t)-c_{2} e_{2}^{2}(t)+e_{2}(t) \xi-e_{2}(t) u_{R} \\
= & -c_{1} e_{1}^{2}(t)-c_{2} e_{2}^{2}(t)-\frac{1}{2} e_{2}^{2}(t)-\frac{1}{2}\left[\frac{e_{2}(t)}{\rho}-\rho \xi\right]^{2} \\
& +\frac{1}{2} \rho^{2} \xi^{2} \leq-\frac{1}{2} e_{2}^{2}(t)+\frac{1}{2} \rho^{2} \xi^{2} .
\end{aligned}
$$

Assume $\xi \in[0, T], \forall T \in[0, \infty)$; then

$$
V_{2}(T)-V_{2}(0) \leq-\frac{1}{2} \int_{0}^{T} e_{2}^{2}(t) d t+\frac{1}{2} \rho^{2} \int_{0}^{T} \xi^{2} d t
$$

When $V(T) \geq 0$, put (37) into (40); it can get

$$
\begin{aligned}
\int_{0}^{T} e_{2}^{2}(t) d t \leq & \mathbf{e}^{\mathrm{T}}(0) \mathbf{e}(0)+\frac{1}{\eta_{1}} \widetilde{\boldsymbol{w}}_{e}^{\mathrm{T}}(0) \widetilde{\boldsymbol{w}}_{e}(0) \\
& +\frac{1}{\eta_{2}} \widetilde{\mathbf{a}}_{e}^{\mathrm{T}}(0) \widetilde{\mathbf{a}}_{e}(0)+\frac{1}{\eta_{3}} \widetilde{\mathbf{b}}_{e}^{\mathrm{T}}(0) \widetilde{\mathbf{b}}_{e}(0) \\
& +\rho^{2} \int_{0}^{T} \xi^{2} d t
\end{aligned}
$$

When the initial condition parameters $\mathbf{e}(0), \widetilde{\boldsymbol{\omega}}_{e}(0), \widetilde{\mathbf{a}}_{e}(0)$, and $\widetilde{\mathbf{b}}_{e}(0)$ are assumed to be zero, the tracking performance of the system is represented as

$$
\int_{0}^{T} e_{2}^{2}(t) d t \leq V_{2}(0)-V_{2}(\infty) .
$$

Thus, according to the Barbalat lemma [18], the output of the system can be gradual tracking command signal and stable.

\section{Simulation Result and Analysis}

The main parameters in the AC system were as follows: the friction coefficient of the system is $B=1.43 \times$ $10^{-4} \mathrm{~N} \cdot \mathrm{m} /\left(\mathrm{rad} \cdot \mathrm{s}^{-1}\right)$; system load rotational inertia: $J=$ $8200 \mathrm{~kg} \cdot \mathrm{m}^{2}$; system load disturbing moment: $93200 \mathrm{~kg} \cdot \mathrm{m}^{2}$; 


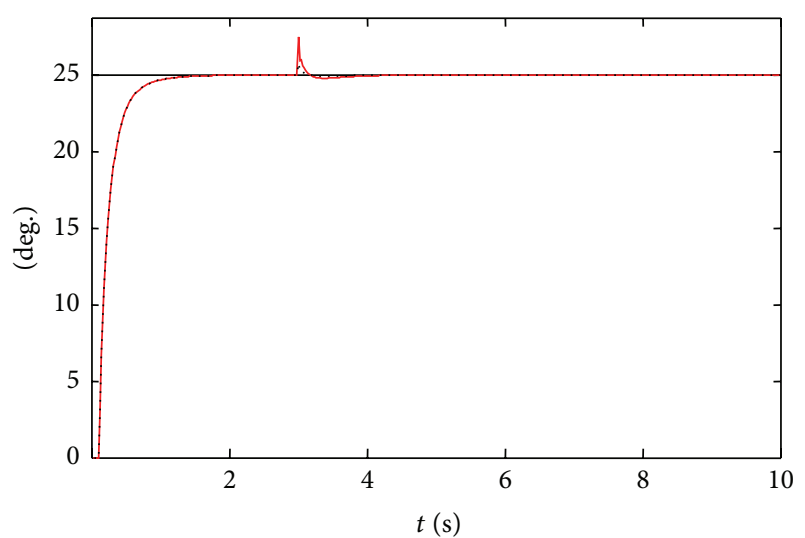

— Reference position

- Adaptive CMAC control

..... SOWCB control

FIGURE 4: Step response curve of load disturbance.

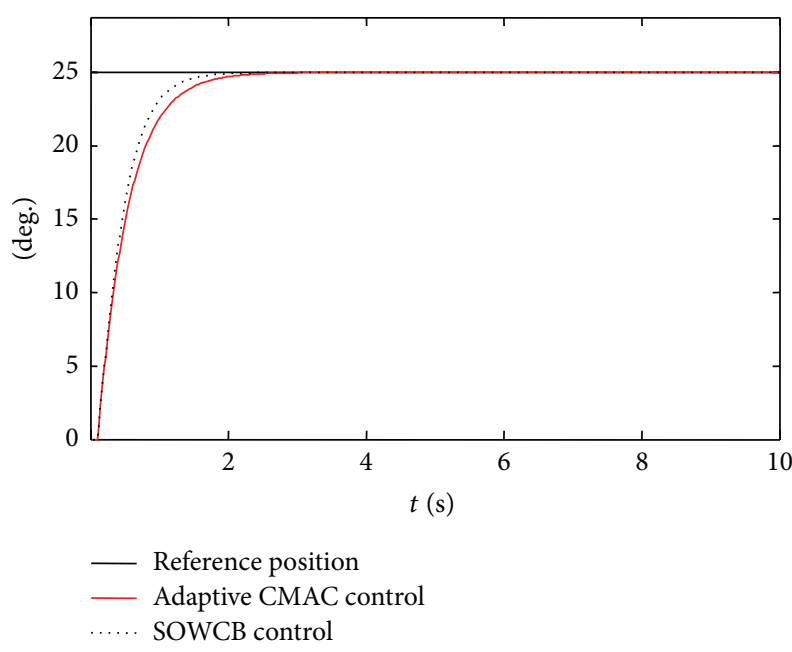

FIgURE 5: The initial moment of inertia of step response curve.

friction moment of the load: $850 \mathrm{~kg} \cdot \mathrm{m}^{2}$; reduction ratio: $i=$ 1002; motor torque coefficient $K_{t}=0.195 \mathrm{~N} \cdot \mathrm{m} / \mathrm{A}$; and choose initial node $n_{b}=1$. The initial parameter is set to the wavelet function: $a_{i 1}=0, b_{i 1}=1.2$. Select input layer in wavelet CMAC $n_{a}=2$; self-organizing wavelet CMAC adaptive backstepping controller parameter selection for $\eta_{1}=0.5$, $\eta_{2}=0.1, \eta_{3}=0.1, \rho=0.2, c_{1}=0.3, m_{\mathrm{th}}=0.5, \beta_{\mathrm{th}}=0.01$, and $\tau=0.01$.

In order to test and verify the effectiveness of the selforganization wavelet adaptive CMAC backstepping control, adaptive CMAC controller is used to compare it. The simulation results are as shown in Figures 4-8.

Figure 4 shows the position response curve added with a $85 \mathrm{Nm}$ step disturbance at $3 \mathrm{~s}$.

As it can be seen from Figure 4, when there is a load disturbance, using CMAC adaptive control algorithm in response to a larger location offset occurs, and it needs $0.83 \mathrm{~s}$ to recover the reference position; however, using selforganizing wavelet adaptive CMAC backstepping control

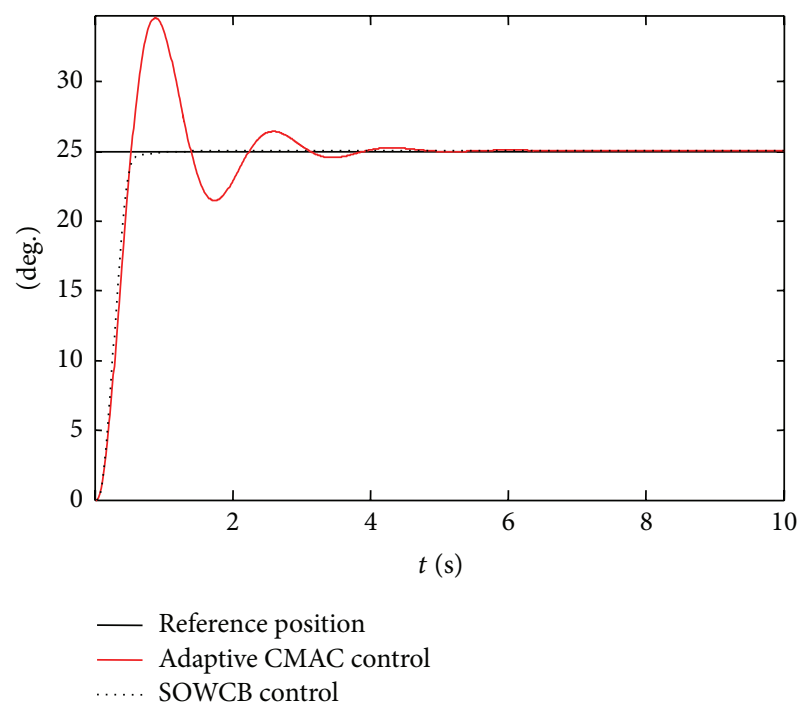

FIGURE 6: The moment of inertia changes step response curve.

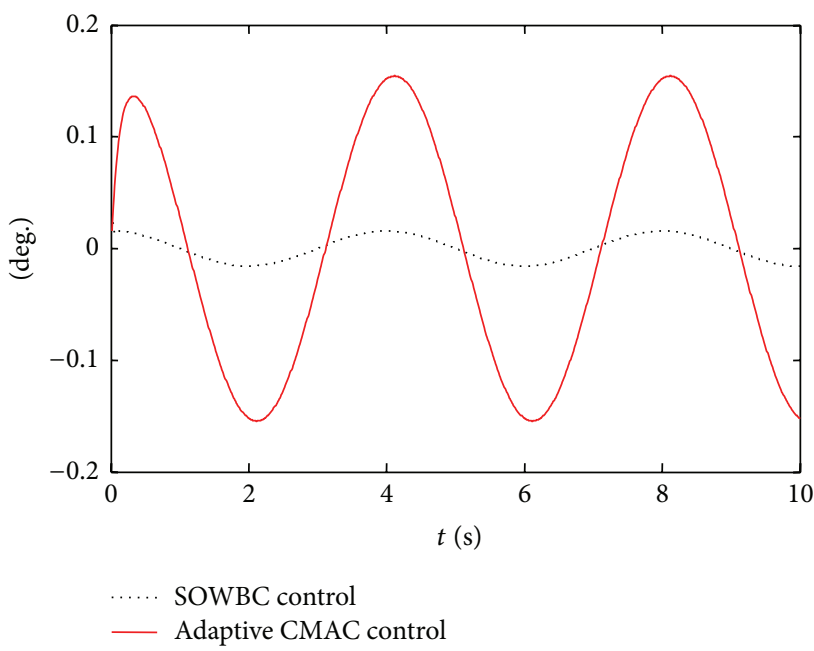

FIgURE 7: Tracking error curve of the system.

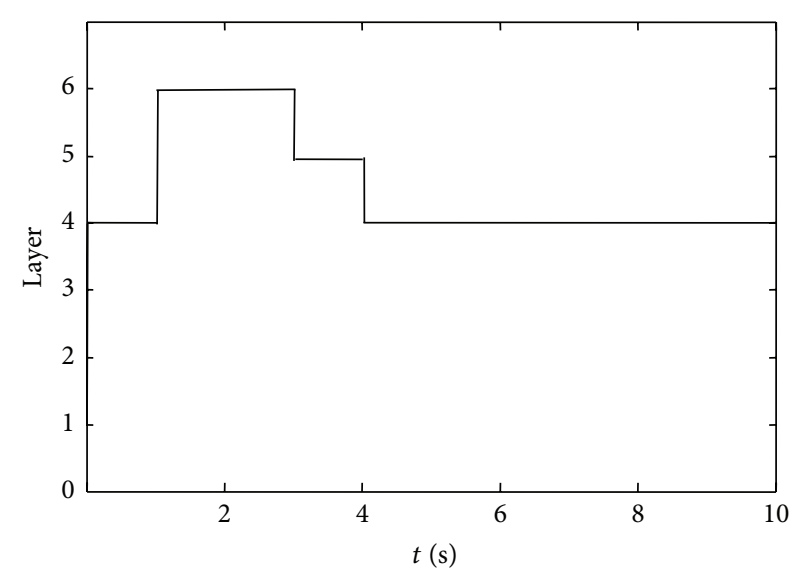

FIgURE 8: The structure of the node curve. 


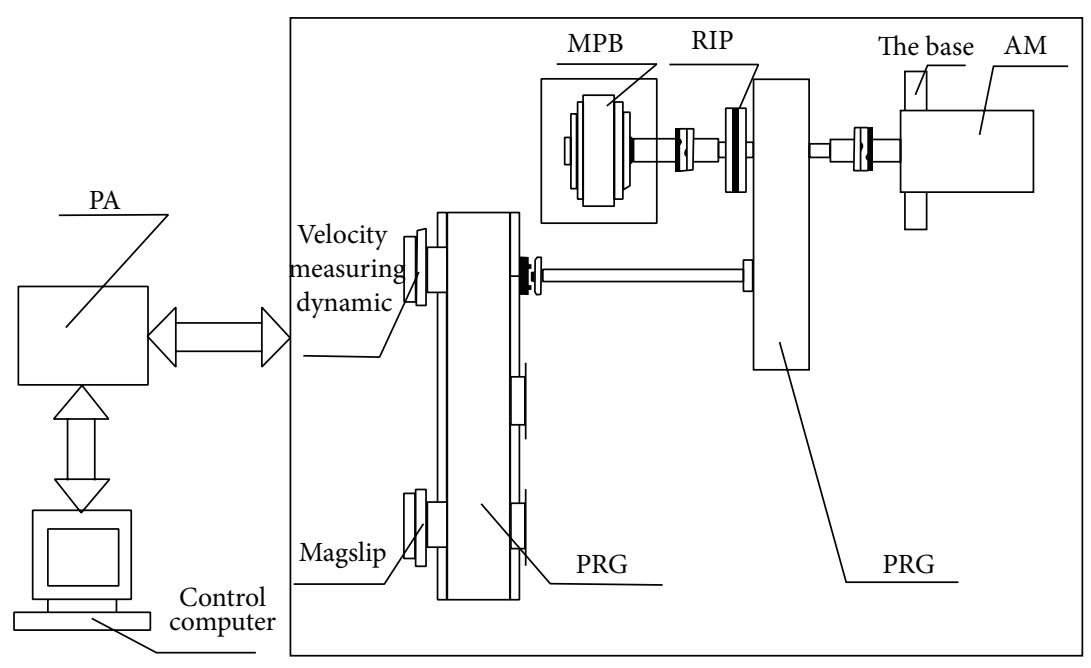

FIGURE 9: Schematic of the semiphysical simulation platform.

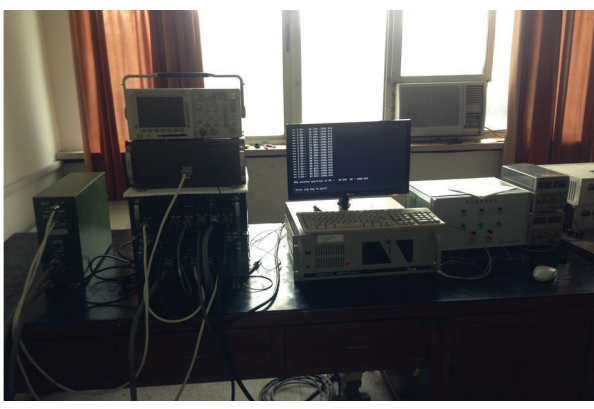

(a)

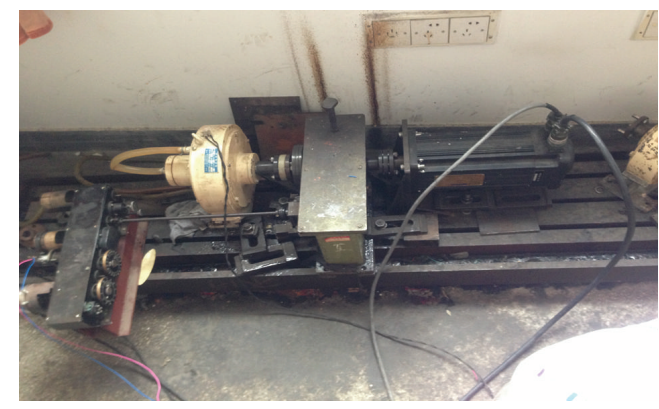

(b)

FIGURE 10: Photograph of the semiphysical simulation platform.

algorithm, the system has better suppression performance of load disturbance, and only $0.45 \mathrm{~s}$ can be stabilized.

Figure 5 shows the position of the initial moment of inertia of the response curve; Figure 6 for the moment of inertia is a variation of the initial value to 1.5 times the position of the response curve.

As can be seen from Figures 5 and 6, when the rotational inertia of the system is as the initial value, there is no overshoot in Figures 5 and 6, using CMAC adaptive control system when the arrival time of the steady state is longer than the required self-organizing adaptive CMAC wavelet backstepping. When the moment of inertia changes, using adaptive CMAC control system to produce the overshoot, and the system required to reach steady state time is $4.52 \mathrm{~s}$, the self-organizing wavelet CMAC adaptive backstepping control is "SOWCB" control, with faster system response time, the time required to reach a steady state is $1.31 \mathrm{~s}$, and the system has better robustness to the change of parameters.

Figure 7 shows a system of sine-input signal tracking error curve; Figure 8 is a node change through selforganization training process from the initial value of 1 to 4 stable nodes.

Figure 7 shows that using adaptive CMAC control system of the maximum tracking error is 0.146 degrees, and, using the self-organizing wavelet CMAC adaptive backstepping control, the maximum tracking error is only 0.0156 degrees. It shows that with the parameter uncertainty in the system and the presence of external disturbances, selforganization designed CMAC adaptive wavelet inversion controller enables the rapid tracking of servo system given position signals and by introducing more efficient robust control suppresses the system impact of various uncertainties, to improve the accuracy and robustness of the system.

\section{Semiphysical Simulation Test}

To investigate the efficiency of the proposed self-organizing wavelet adaptive CMAC backstepping control as a strategy in establishing AC servo system, a semiphysical simulation platform is constructed to simulate the working conditions of the servo control system. The test results were compared to verify the performance of the controller in this paper superiorly.

The semiphysical simulation test-bed structure diagram and object diagram are as shown in Figures 9 and 10, respectively. Based on the components shown in Figure 9, the platform consists of seven parts, including the control computer, the sensor system for measurement, the power 


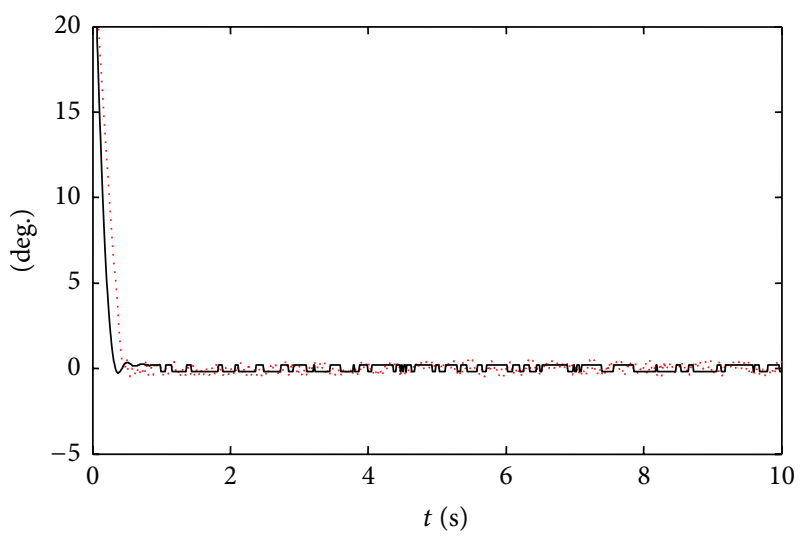

Adaptive CMAC control

— SOWCB control

FIGURE 11: System step response tracking error.

amplifier (PA), the precision reduction gearbox (PRG), the loading fixture (LF), the actuating motor (AM), and the test bed. The loading fixture, which consists of the rotational inertia plate (RIP) and the magnetic powder brake (MPB), is employed for the simulation of the rotational inertia, the load torque, and the frictional resistance moment. The rotational inertia variations in the loads are well simulated by changing the RIP. Similarly, the variations in the load torque and the frictional resistance moment are also well simulated by controlling the output torque of the MPB.

To investigate the tracking accuracy of the servo system with adaptive self-organizing wavelet CMAC backstepping control system, sinusoidal command tracking with a frequency of $1 \mathrm{~Hz}$ and amplitude of 100 degrees is conducted on the semiphysical simulation platform. The corresponding tracking errors of both the SOWCB and adaptive CMAC control systems are illustrated in Figure 11.

The figure also illustrates that the SOWCB control system has a smaller steady-state error and external disturbance error showed stronger inhibitory action and has faster response speed and good robustness.

\section{Conclusions}

In this paper, due to the existence of nonlinear servo system problem, self-organizing CMAC adaptive wavelet backstepping control methods have been proposed. The simulation and prototype test results showed that

(1) compared to self-organization wavelet algorithm with the traditional CMAC method, it has higher accuracy;

(2) the scheme of system uncertainties and external disturbance has strong robustness and good dynamic and steady-state response performance.

\section{Conflict of Interests}

The authors declare that there is no conflict of interests regarding the publication of this paper.

\section{References}

[1] J. Zhou and Y. Wang, "Adaptive backstepping speed controller design for a permanent magnet synchronous motor," IEE Proceedings: Electric Power Applications, vol. 149, no. 2, pp. 165172, 2002.

[2] S. Zhu, M. X. Sun, and X. X. He, "Iterative learning control of strict-feedback nonlinear time-varying systems," Acta Automatica Sinica, vol. 36, no. 3, pp. 454-458, 2010.

[3] Y.-P. Sun, J.-M. Li, and J.-A. Wang, "Adaptive learning control of nonlinear systems with iteration-varying trajectory," Systems Engineering and Electronics, vol. 31, no. 7, pp. 1715-1719, 2009.

[4] X.-L. He and S.-C. Tong, "Direct adaptive fuzzy backstepping control of nonlinear systems with dynamic uncertainties," Control Theory and Applications, vol. 26, no. 10, pp. 1081-1086, 2009.

[5] C.-F. Hsu, C.-M. Lin, and T.-T. Lee, "Wavelet adaptive backstepping control for a class of nonlinear systems," IEEE Transactions on Neural Networks, vol. 17, no. 5, pp. 1175-1183, 2006.

[6] D. Liu and M. Li, "PMSM position servo control based on wavelet neural network adaptive backstepping," Electric Power Automation Equipment, vol. 33, no. 2, pp. 126-130, 2013.

[7] C.-M. Lin, K.-N. Hung, and C.-F. Hsu, "Adaptive neuro-wavelet control for switching power supplies," IEEE Transactions on Power Electronics, vol. 22, no. 1, pp. 87-95, 2007.

[8] J. S. Albus, "A new approach to manipulator control: the cerebellar model articulation controller (CMAC)," Journal of Dynamic Systems, Measurement and Control, vol. 97, no. 3, pp. 220-227, 1975.

[9] C.-M. Lin and Y.-F. Peng, "Adaptive CMAC-based supervisory control for uncertain nonlinear systems," IEEE Transactions on Systems, Man, and Cybernetics, Part B: Cybernetics, vol. 34, no. 2, pp. 1248-1260, 2004.

[10] Y.-F. Peng, "Robust intelligent backstepping control system using RCMAC for tracking periodic trajectories," Nonlinear Analysis: Real World Applications, vol. 12, no. 3, pp. 1371-1385, 2011.

[11] C.-M. Lin, L.-Y. Chen, and C.-H. Chen, "RCMAC hybrid control for MIMO uncertain nonlinear systems using slidingmode technology," IEEE Transactions on Neural Networks, vol. 18, no. 3, pp. 708-720, 2007.

[12] C. T. Ching and C. S. Lin, "CMAC with general basis functions," Neural Networks, vol. 9, no. 7, pp. 1199-1211, 1996.

[13] H.-M. Lee, C.-M. Chen, and Y.-F. Lu, "A self-organizing HCMAC neural-network classifier," IEEE Transactions on Neural Networks, vol. 14, no. 1, pp. 15-27, 2003.

[14] C.-M. Lin and H.-Y. Li, "Self-organizing adaptive wavelet CMAC backstepping control system design for nonlinear chaotic systems," Nonlinear Analysis: Real World Applications, vol. 14, no. 1, pp. 206-223, 2013.

[15] L. Wu, X. Su, P. Shi, and J. Qiu, "Model approximation for discrete-time state-delay systems in the T-S fuzzy framework," IEEE Transactions on Fuzzy Systems, vol. 19, no. 2, pp. 366-378, 2011.

[16] Y.-G. Leu, T.-T. Lee, and W.-Y. Wang, "Observer-based adaptive fuzzy-neural control for unknown nonlinear dynamical systems," IEEE Transactions on Systems, Man, and Cybernetics, Part B: Cybernetics, vol. 29, no. 5, pp. 583-591, 1999.

[17] W. Zhang, W. Liu, X. Ye, Y. Zhu, and X. Hu, "Robust adaptive control for free-floating space manipulators based on neural 
network," Journal of Mechanical Engineering, vol. 48, no. 21, pp. 36-40, 2012.

[18] Y.-M. Fang, S.-C. Ren, Z.-J. Wang, and X.-H. Jiao, "Adaptive fuzzy backstepping control for speed of permanent magnet synchronous motor," Electric Machines and Control, vol. 15, no. 6, pp. 97-102, 2011. 


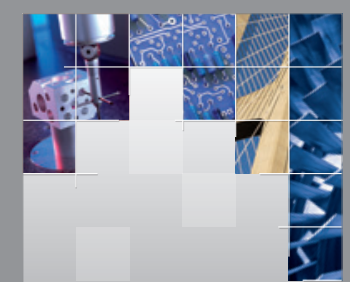

\section{Enfincering}
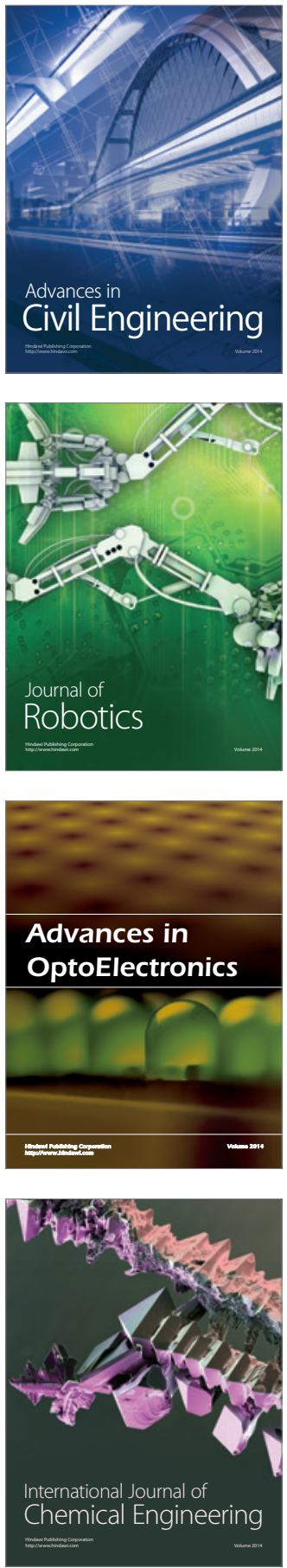

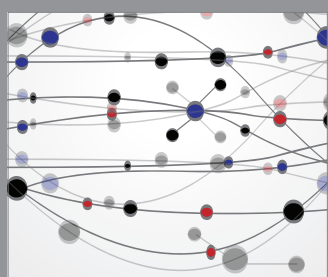

The Scientific World Journal

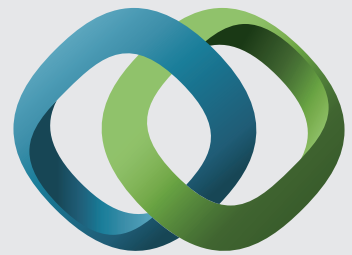

\section{Hindawi}

Submit your manuscripts at

http://www.hindawi.com
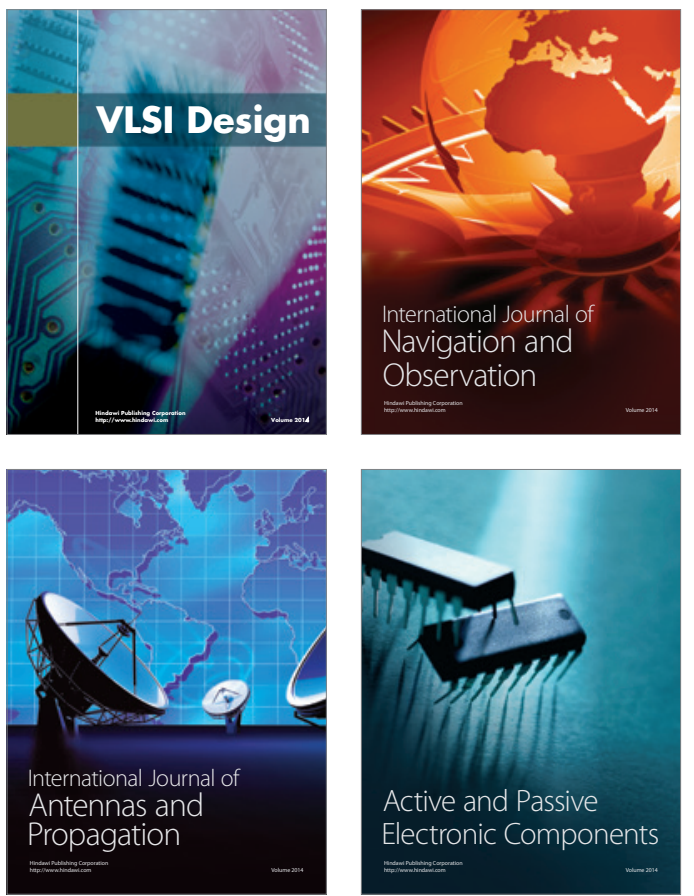
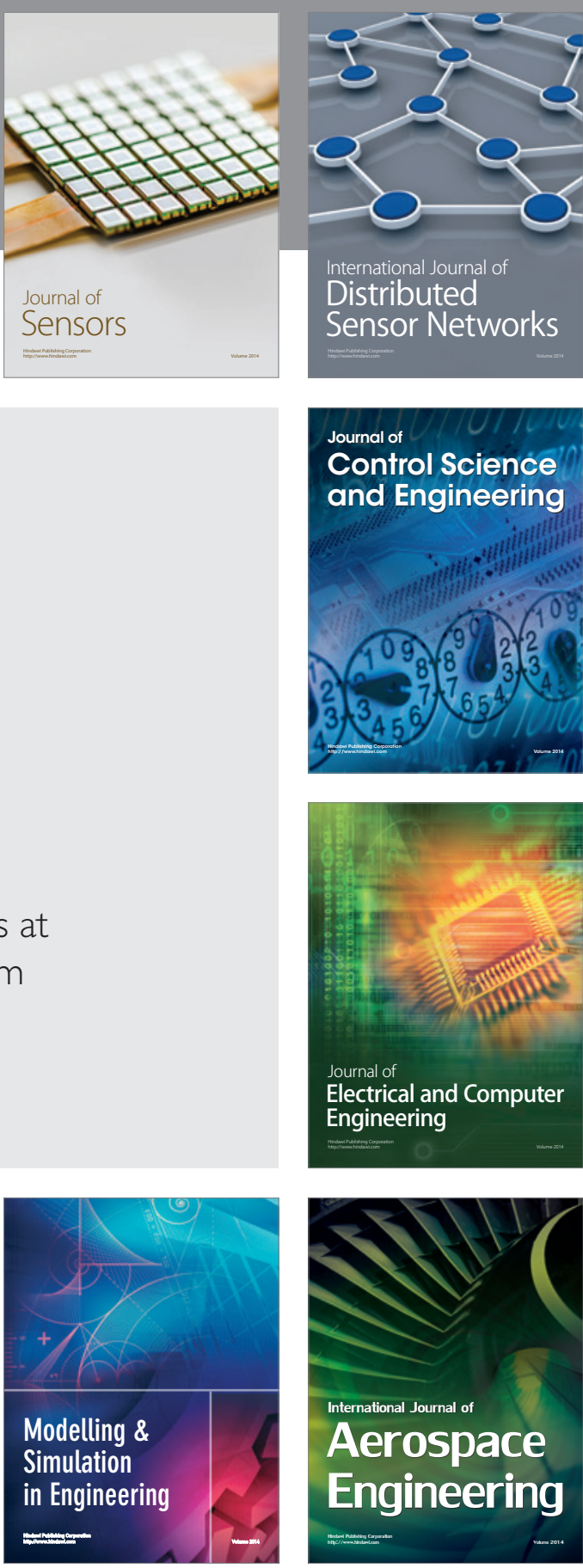

International Journal of

Distributed

Sensor Networks

Journal of

Control Science

and Engineering
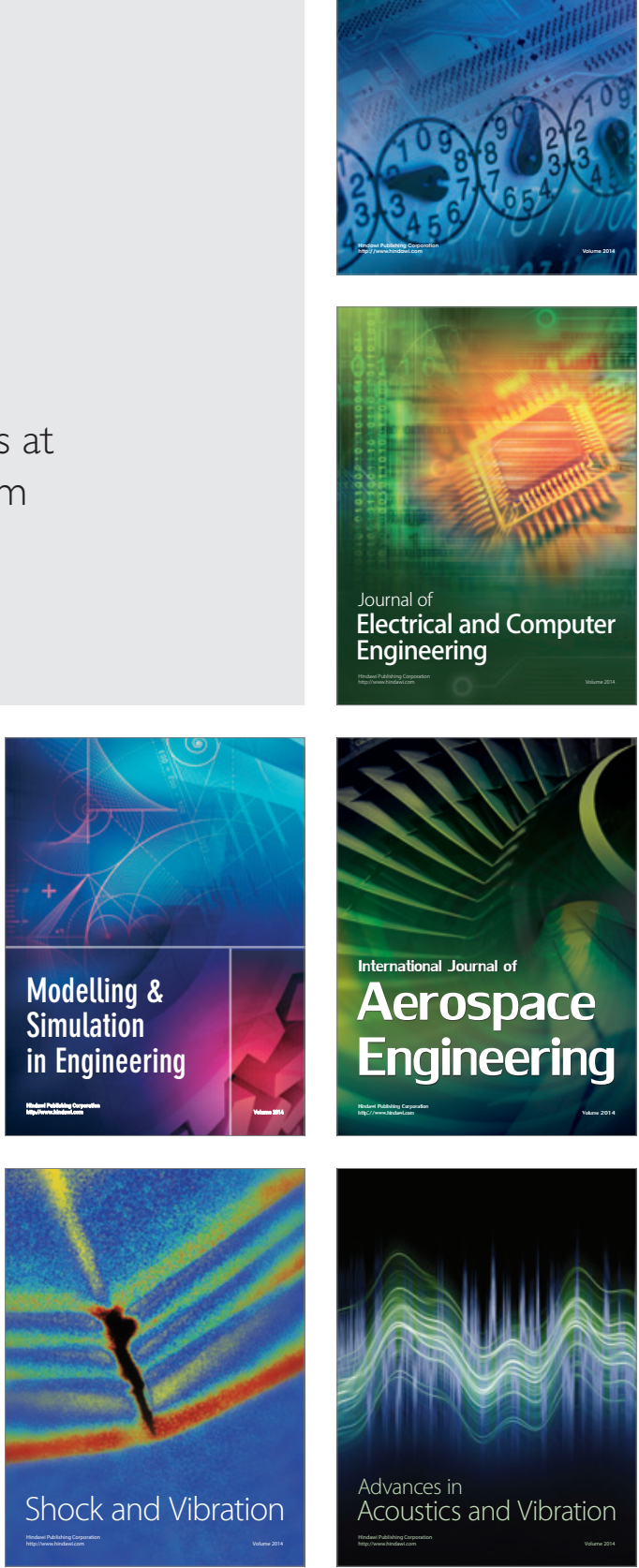